\title{
"Comme c'est un peu langage des jeunes quoi »: analyse du marqueur discursif quoi dans le discours des Chinois résidant en France
}

\author{
Delin Deng* \\ École des Hautes Études en Sciences Sociales, Anthropologie Sociale et Ethnologie
}

\begin{abstract}
Résumé. Il est documenté que dans les décennies récentes quoi commence à être utilisé comme marqueur discursif dans le discours des locuteurs natifs du français. Cependant, cet usage n'est mentionné ni dans le dictionnaire ni dans les méthodes de grammaire. Malgré des travaux fructueux à la fois qualitatifs et quantitatifs sur sa fonction syntactique et discursive dans le discours des locuteurs natifs du français, sa présence dans le discours des locuteurs non-natifs n'attirent pas encore beaucoup d'attention. Cet article a donc pour objectif de comparer l'usage du marqueur discursif quoi dans le discours des locuteurs non-natifs avec celui dans le discours des locuteurs natifs. Nous nous concentrons sur les divers contextes sous lesquels il apparait ainsi que comment des facteurs sociaux influencent son emploi. En conclusion, on constate que les locuteurs non-natifs utilisent quoi beaucoup moins fréquemment que les locuteurs natifs. Cependant, ils l'emploient sous des contextes différents tout en tenant compte que les contextes sont plus complexes et variés chez les locuteurs natifs. En ce qui concerne les facteurs extralinguistiques, les hommes utilisent beaucoup plus de quoi que les femmes. La durée de séjour et le statut professionnel semblent aussi significatifs pour son emploi dans notre étude.
\end{abstract}

\begin{abstract}
Analysis of discourse markers quoi in the speech of French L2 speakers: the case of the Chinese living in Paris. It is observed that quoi has started to be used as discourse marker in French native speakers' speech in the recent decades. However, this usage is not mentioned neither in the dictionary nor in the grammar books. Despite of some fruitful studies in this usage in French native speakers' speech, its appearance in non-native speakers' speech, to our knowledge, has not yet drawn enough attention. Therefore, this article aims to compare the use of the discursive marker quoi in the speech of nonnative speakers of French with that in the speech of native speakers of French, in particular the various contexts under which this discursive marker appears and how social factors influence its usage. In conclusion, we find that these speakers not only learnt to use quoi as a discursive marker but also in different contexts. However, the contexts are more complex and varied in the speech of the native speakers. As to the extralinguistic factors, male speakers use much more quoi than female speakers. Length of stay and employment status also seemed to be significant for its usage in our study.
\end{abstract}

\footnotetext{
*Corresponding author : delin.deng@gmail.com
} 


\section{Introduction}

L'objectif de cet article est de voir dans un premier temps si les locuteurs non-natifs du français utilisent aussi quoi comme marqueur discursif dans leur production orale. Cet usage comme marqueur discursif est assez récent chez les locuteurs natifs du français et représente, selon Beeching (2007), un parler des jeunes locuteurs masculins. Cet emploi a souvent une connotation péjorative, considéré comme mal parler. D'après Chanet (2001), quoi apparaît régulièrement sous des contextes de bafouillage. Elle a ensuite identifié quatre fonctions discursives de quoi. Lefeuvre (2011) a indiqué dans son article que quoi apparaît surtout quand la formulation est problématique. Elle a montré cinq cas pour sa présence dans un énoncé. Par conséquence, nous allons montrer comment une telle classification est lacunaire et donc le rend impossible de prévoir la présence de quoi dans un énoncé purement basé sur des marqueurs saillants. Nous nous concentrerons, dans un deuxième temps, sur l'emploi de quoi dans le discours des locuteurs non-natifs. Dernièrement, comme quoi est un marqueur socialement marqué et que son usage est influencé par des facteurs sociaux, tels que l'âge, le sexe, la classe sociale et le niveau d'éducation du locuteur, nous souhaitons de voir si cela est aussi le cas pour les locuteurs non-natifs.

Pour cet article, nous partirons des «petits mots » sur lesquels s'appuie le discours tels que fin, quoi, voilà, etc. Ces petits mots portent en fait beaucoup de noms : "marqueurs discursifs » (voir Schiffrin, 1988, Jucker \& Ziv, 1998, Fraser, 1999, Dostie, 2004 et Andersen, 2007), « marqueurs pragmatiques »(Erman, 2001), «particule énonciative » (Fernandez, 1994), « discourse particle » (Schorup, 1985 et Hansen, 1997), « pragmatic particle » (Beeching, 2002), « opérateur discursif » (Redeker, 1990, 1991) ou « discourse connective » (Blakemore, 1987). La variété de la terminologie représente plutôt une diversité de la préoccupation dans la fonction des marqueurs chez des linguistes différents qui travaillent dans des domaines différents. Ici, nous prenons plutôt le terme « marqueur discursif » pour le reste de cet article.

Dans une première partie, les travaux précédents sur le marqueur discursif quoi dans le parler des locuteurs natifs du français seront revisités. Ensuite, dans une deuxième partie, tout en s'appuyant sur les données de notre propre corpus recueillies auprès des Chinois de la première génération résidant en France suivant la méthodologie sociolinguistique, nous allons présenter l'usage du marqueur discursif quoi dans le parler de nos locuteurs non-natifs du français. Nous mettrons en évidence que le marqueur discursif quoi est utilisé presque de la même manière dans le discours des locuteurs non-natifs que dans le parler des locuteurs natifs. Parallèlement, nous examinons les trois facteurs sociaux qui pourraient être pertinents pour son emploi chez les locuteurs non-natifs : le sexe, la durée de séjour et le statut professionnel. Pour conclure, nous posons une réflexion sur l'apprentissage des marqueurs discursifs au sein de la classe des langues ainsi qu'une éventuelle stigmatisation des façons de parler.

\section{Revue de littérature}

Chanet (2004) a établi une liste des fréquences des 85 marqueurs discursifs par les locuteurs natifs du français en France dans leur production orale. Quoi occupe la huitième place sur cette liste avec une fréquence d'emploi de 1 occurrence toutes les 1 minute 53 secondes. Il est clair que quoi est un des marqueurs les plus fréquents dans le discours des natifs. Étant donné sa popularité dans son usage quotidien, il est surprenant de voir les études peu nombreuses sur son emploi. En tant que marqueur discursif, quoi ne rend plus dans la catégorie morphologique qui lui est attribuée. En fait, il «perd ses propriétés de pronom lorsqu'il devient particule » (Chanet, 2004). C'est-à-dire que si quoi est pronom, il ne pourrait pas être un marqueur discursif, et vice versa.

Après une analyse des 1700 occurrences de la particule quoi en français parlé contemporain, Chanet (2001) a montré que quoi sont employés dans les trois statuts suivants dans les corpus de français parlé avec le pourcentage des occurrences exploitables entre parenthèses : 
1) pronom $(16.56 \%)$

2) locution figée $(4.71 \%)$

3) particule énonciative (76.31\%)

D'après cette distribution, on pourrait voir qu'environs trois quarts de quoi sont utilisés comme marqueur discursif par les locuteurs natifs du français dans leur production orale. Cela suggère également que quoi perd de plus en plus son statut morphologique comme pronom en français parlé et pourrait passer dans un blanchissement sémantique, qui lui attribue de nouvelles fonctions discursives.

En ce qui concerne sa fonction, d'après Chanet (2001), il y a principalement quatre fonctions discursives:

1) Quoi indique qu'il y a eu une évaluation, de la part du locuteur, de sa propre production, et des représentations susceptibles d'être mentalement construites à partir de cette production.

2) Quoi indique que le locuteur peut douter du caractère «suffisant » des informations qu'il donne pour la reconstruction de la schématisation par l'allocutaire.

3) Quoi signale qu'un ensemble de possibles énonciatifs et cognitifs peut être ouvert là où il apparaît, possibles qui contribueraient à permettre une reconstruction de la schématisation par l'allocutaire, s'il les imaginait.

4) Quoi constitue une invitation (à destination de l'allocutaire) à reconstruire la schématisation en tenant compte du positionnement du locuteur parmi ces possibles, c'est-à-dire une invitation à partager des représentations nécessairement subjectives en effectuant des inférences.

Selon cette analyse, le marqueur discursif quoi est "une particule de connivence». (Beeching, 2007)

Beeching (2002) a remarqué que dans son corpus, « la majorité des occurrences marque une hésitation par rapport au caractère adéquat de l'expression $(80 \%)$ ». Le quoi « sépare le dire et le dit, indiquant une gêne par rapport au caractère adéquat de l'expression ». Elle indique aussi que quoi n'est pas très valorisé du point de vue sociolinguistique. Cependant, quoi «convient très bien à des formes de politesse moins formelles axées sur la solidarité ». Elle confirme également ce qui est dit dans l'étude de Chanet (2004) que les locuteurs masculins emploient le quoi davantage que les locuteurs féminins.

Lefeuvre $(2006,2011)$ a mentionné dans ses recherches que l'usage du marqueur discursif quoi signifie une recherche de formulation. Par conséquence, il apparaît régulièrement quand la formulation est problématique. Elle a suggéré les cinq cas suivants pour la présence du marqueur discursif quoi (les exemples 1-5 sont de Lefeuvre):

1) après une répétition des segments de l'énoncé:

(1) spk1:non+non++pas du tout pas du tout non donc la petite bande du lycée c'est pas c'est pas votre votre vie quoi

spk2 : et j'm'en rends compte qu'on croyait qu' c'était classique et c'était habituel et c'était pas habituel du tout hein

spk1 : oui euh euh la plupart des familles (CFPP2000, [07-03])

2) après des marqueurs d'hésitation tels que euh:

(2) $\operatorname{spk} 3$ : euh on y va on y va doucement (rires) justement l'idée c'était + je savais pas exactement où il était + euh mais on allait $\mathrm{y} a-+$ on allait $\mathrm{y}$ aller à pieds et euh ça nous ferait faire une belle balade quoi (CFPP2000, [KB-01])

3) après des marqueurs d'approximation tels que comme ça

(3) spk1 : oui + j'ai vu de l'absentéisme euh des + + une amie à moi qu'était vraiment paniquée euh son gamin fuguait pour aller pour ne pas aller euh à l'école donc passait ses journées à s'promener alors il avait quoi c'était l'année dernière il était en cinquième + parce que euh un en 
français deux en histoire géo trois en maths avec des moyennes comme ça donc quoi alors (CFPP2000, [11-01])

4) après des segments de positionnement tels que je trouve :

(4) spk2: oui + donc vous êtes sévère pour le collège

spk1 : oui j'trouve qu'il y a beaucoup d'oralité quoi c'est vraiment euh

j'trouve que le (CFPP2000, [11-01])

5) avec des paradigmes de choix possibles de formulation:

(5) et puis après bah non euh: c'était toute la période:: c'est surtout Brigitte Bardot qui m'a frappée à c't'époque là et puis euh: Françoise Arnoux euh Charles Boyer des gens comme ça quoi (CFPP2000, [11-02])

On pourrait bien voir que cette classification en fait correspond partiellement aux quatre fonctions discursives de quoi proposées par Chanet (2001). Néanmoins, il semble que cette classification pose plusieurs problèmes. D'abord, la différence entre l'exemple 3 et l'exemple 5 n'est pas très clarifiée. En fait, ce que Lefeuvre appelle «marqueur d'approximation » comme ça dans l'exemple 3 fonctionne plutôt comme une particule d'extension qui généralise le dit. À part de ce problème d'appellation, dans les deux exemples, nous avons vu la même particule d'extension comme ça. Si l'exemple 3 considère que l'apparition de quoi est liée à la présence de la particule comme ça, qu'est-ce qui rend l'exemple 5 différent de l'exemple 3 ? En fait, dans les deux cas, quoi clore l'énumération et indique un ensemble de possibles énonciatifs.

Un autre problème est que cette classification est basée sur des marqueurs saillants dans l'énoncé pour prévoir la présence de quoi et par conséquence ne couvre pas tous les contextes. Certains cas ne pourrait pas être expliqués par cette liste. Nous utilisons l'exemple suivant de Chanet (2001) pour illustrer cette idée :

(6) L1 [...] ça c'est tous les testeurs hein ce n'est pas pas grandeur nature

L3 mh ah oui c'est oui

L1 oui oui voilà c'est les m-c'est les c'est les trucs de démonstration hein non parce qu'en

L2 non il y a des jolies couleurs

L1 nature là ils font trois millilitres ils en font douze donc ils sont quatre fois plus grands hein en en dimension réelle

L2 comme ceux que l'on achète dans le commerce quoi non

L1 ben je vais te montrer je dois en avoir un que qui est $\mathrm{X}$ là (817, BEAUT § 186)

Il est évident que l'exemple 6 ne rend pas dans les cinq cas susmentionnés. Il n'y a ni de répétition, ni de marqueur d'hésitation, ni de segments de positionnement, ni de paradigmes de choix. Cependant, le quoi ici constitue une invitation à reconstruire la schématisation tout en faisant appel à un stéréotype. Il s'agit dans ce cas-là une simple invitation à partager des connaissances.

Jusqu'ici, il semble impossible de prévoir la présence de quoi purement d'après des indications saillantes, puisqu'il y a des cas où on ne voit pas des marqueurs comme je trouve ou une répétition sur un même syntagme. Il vaut mieux peut-être classer les cas pour sa présence selon sa propre fonction discursive.

\section{Méthodologie}

Dans cette partie, nous allons présenter les détails sur le corpus, les locuteurs et les facteurs extralinguistiques dans l'étude présente.

\subsection{Corpus et données}


Les données du corpus dont nous nous servons ont été collectées entre 2014 et 2015 en France : Les analyses présentées portent sur le sous-corpus de conversations semi-dirigées en français recueilli auprès de 37 locuteurs et comptant un total de 27 heures d'enregistrement.

La conversation varie de 30 minutes à 1 heure 30 minutes en fonction du locuteur. Pour cette tâche, nous avons préparé avant l'interview une liste de questions à poser au cours de l'interview. Cependant, il faut tenir compte que l'interview n'a pas été réalisée comme un simple aller-retour de question-réponse entre l'intervieweuse et l'interviewé. Ces questions étaient davantage des supports pour que l'interviewé puisse raconter librement ses expériences. Toutes les interviews ont été enregistrées et transcrites entièrement. Puisque les locuteurs dans notre corpus sont des locuteurs non-natifs, on trouverait des erreurs grammaticales dans leur production orale. Pour la transcription, nous n'avons pas fait la correction. Toutes les interviews ont été transcrites telles qu'elles ont été produites.

Toutes les occurrences de quoi dans notre corpus ont été relevées et analysées.

\subsection{Locuteurs}

Les 37 locuteurs sont tous d'origine chinoise et résidaient en France au moment de l'interview. Ils parlent tous le français en tant que langue seconde. Ces locuteurs ont été recrutés par le réseau social de l'intervieweuse et des interviewés avec la technique de boule de neige.

Ces locuteurs, dont 22 femmes et 15 hommes, sont âgés de 20 ans à 55 ans. Ils n'ont pas les mêmes compétences linguistiques en français. Leur exposition à la langue est différente en fonction de leur durée de séjour en France. Certains locuteurs vivent en France depuis plus de dix ans. Ils sont donc plus intégrés à la communauté locale et à l'environnement social en général. D'autres viennent d'arriver en France sous un an.

Enfin, le statut professionnel de nos locuteurs sont différents. Ceux qui mènent une vie professionnelle ont plus de contacts avec les Français au travail et après le travail et ont donc plus d'opportunités de pratiquer le français quotidiennement. Les étudiants travaillent plutôt à temps partiel, pour la plupart dans des restaurants chinois ou japonais, tenus par des patrons chinois. Ainsi, même s'ils travaillent, ils ne pratiquent pas vraiment la langue durant leurs journées de travail. Cela pourrait donc avoir une influence sur leur usage du français.

\subsection{Facteurs extralinguistiques}

Comme l'évoqué plus haut, l'emploi du marqueur discursif quoi est influencé par des facteurs sociaux, dit aussi des facteurs extralinguistiques, tels que l'âge, le sexe, le niveau d'éducation, la classe sociale du locuteur. Pour nos locuteurs non-natifs, nous examinons les facteurs suivants : le sexe, la durée de séjour et le statut professionnel.

Étant donné que nous avons 22 locuteurs féminins contre 15 locuteurs masculins, le nombre inégal des membres dans ces deux groupes de sexe pourrait poser des problèmes pour l'interprétation des résultats seulement si les locuteurs féminins produisent plus d'occurrences de quoi que les locuteurs de sexe opposé. Dans le cas inverse, le résultat correspond alors à celui chez les locuteurs natifs.

Ensuite, la durée de séjour semble aussi un facteur pertinent dans le cas des locuteurs nonnatifs. Pour notre analyse, nous les avons divisés en trois groupes : ceux qui ont passé 1-3 ans en France, ceux qui ont vécu 4-6 ans en France et ceux qui ont résidé plus de 7 ans en France. Nous supposons que la plus longue la durée de séjour, le plus on utilise quoi.

Enfin, puisque le différent statut professionnel leur donne des accès différents au registre informel du français parlé et que le registre informel favorise l'usage des marqueurs discursifs, cela pourrait également avoir une influence sur leur emploi de quoi. Notre hypothèse est que ceux qui mènent une vie professionnelle utilisent plus de quoi dans leur discours. 


\section{Marqueur discursif quoi dans le discours des locuteurs non-natifs du français}

\subsection{Tendance générale}

Dans notre corpus, contrairement à son occurrence abondante chez les locuteurs natifs, nous ne trouvons pas beaucoup d'occurrences de quoi dans la production orale de nos locuteurs nonnatifs. Nous n'avons relevé que 272 occurrences de quoi sur 27 heures d'interview, dont 165 ont été utilisés comme marqueur discursif, soit une fréquence d'emploi de 1 occurrence toutes les 9 minutes 48 secondes. Comparé à sa fréquence d'emploi chez les locuteurs natifs selon la liste de Chanet (2004), soit 1 occurrence toutes les 1 minute 56 secondes, les locuteurs nonnatifs dans notre corpus l'utilisent beaucoup moins fréquemment. Lorsque $76 \%$ des quoi sont employés comme marqueur discursif dans le discours des locuteurs natifs, cette proportion chez les locuteurs non-natifs est d'environs $60 \%$.

\subsection{Emploi de quoi dans le discours des locuteurs non-natifs du français}

Jusqu'ici il est clair que les locuteurs non-natifs emploient quoi comme marqueur discursif dans leur discours, néanmoins beaucoup moins fréquemment que les locuteurs natifs. Comme nous avons déjà montré dans la partie précédente, dans la production orale des locuteurs natifs, quoi a des fonctions discursives diverses. Selon le modèle de Chanet (2001), il y a cinq cas pour l'apparition de quoi dans le discours: processus de production et de planification; référenciation, catégorisation et partage de connaissance; quantification, graduation et construction de l'intersubjectivité ; positionnements énonciatifs ; concession et argumentation. Ce modèle est basé sur la fonction discursive de quoi, et par conséquence, plus complète que le modèle proposé par Lefeuvre. La question que nous voulons poser ici est si quoi est utilisé aussi avec une variété de fonctions discursives dans le discours des locuteurs non-natifs ? Dans cette partie, nous regardons en particulier si nos locuteurs non-natifs emploient quoi dans ces cinq cas susmentionnés.

\subsubsection{Marqueur de planification}

Quoi pourrait fonctionner d'abord comme un marqueur de planification, qui nous montre les tentatives du locuteur dans sa production orale. Très souvent, cela ressemble beaucoup à un bafouillage, notamment quand le locuteur piétine sur un même syntagme. Pour les locuteurs non-natifs, une telle répétition pourrait être perçue comme un manque de fluidité. Il correspond au premier cas de Lefeuvre :

(7) euh peut-être c'était nécessaire peut-être pas mais j'ai commencé un peu de um cours pendant deux mois c'était deux cours um chacun durait un mois ça c'est le base prononciation comme c'est c'est c'est débutant quoi mais dès que je suis venue ici mon niveau c'était fauxdébutant voilà (2015, YW)

Il est clair qu'ici le locuteur piétine sur le segment «c'est». Cette répétition montre une recherche de formulation. Cependant, la répétition n'est pas une nécessité préalable pour la présence de quoi. Il y a aussi des cas où on ne trouve pas de répétition, mais plutôt une reformulation :

(8) c'est pas La/c'est pas Latin en fait c'est vraiment tradition la tradition fin tu vois les lettres c'est pas mais par contre en Turquie leur leur président euh fin le système est latinisé je sais pas XXX sont les lettres sont Latin quoi $(2015, \mathrm{JWH})$

Souvent la reformulation donne à une précision. Comme dans l'exemple 8 , le locuteur commence par un terme plus général « les lettres » et finit par un terme plus précis « Latin ». 


\subsubsection{Marqueur de référenciation}

Quand quoi est employé comme un marqueur de référenciation, on trouve souvent comme dans un même énoncé que quoi pour mettre en comparaison à une connaissance que le locuteur suppose que son allocutaire partage.

(9) c'est comme ce qu'on a appris en Chine mais avec un professeur français quoi voilà (2014, MWL)

Certes, la comparaison n'est pas faite exclusivement en faisant appel à l'emploi de comme. Il pourrait être des particules d'extension, tels que quelque chose comme ça. Dans ce cas-là, quoi clore l'énumération. Le locuteur invite son allocutaire à reconstruire la schématisation. C'est le cas de l'exemple 3 et 5 de Lefeuvre. Cependant, nous ne trouvons pas d'occurrence de ce type dans notre corpus.

Selon le modèle de Chanet, une référenciation nécessite pas l'usage d'une comparaison, mais l'emploi des expressions référentielles générique. Nous ne trouvons non plus l'exemple de ce type dans notre corpus.

\subsubsection{Marqueur de quantification}

Pour les locuteurs natifs, il y a plusieurs quantificateurs qui pourraient s'employer avec quoi, tels que tous les, jamais, assez, etc. Dans notre corpus, le quantificateur le plus fréquent est un peu :

(10)en fait souvent euh un emploi c'est un terme pour les gens qui habitent dans ce banlieue un peu populaire quoi $(2015, \mathrm{FSJ})$

On peut constater qu'avec l'emploi de $u n ~ p e u$, le locuteur invite son allocutaire à partager son positionnement tout en rendant compte que ce positionnement pourrait être subjectif.

\subsubsection{Marqueur de positionnement}

Le cas le plus souvent avec cet usage est la présence de je trouve, je pense dans l'énoncé, qui correspond au quatrième cas de Lefeuvre :

(11)moi je peux pas je peux pas souffrir parce que je pense que c'est la France quoi il est quelqu'un qui pauvre qui peux prend les choses comme ça (2014, LZF)

Cependant, quoi peut aussi apparaître avec un vraiment évaluatif :

(12) mais pour les débutants bon c'est vraiment euh je sais pas nightmare quoi donc $\mathrm{j}$ 'ai jamais réussi à distinguer les temps différents et en plus il y a aussi et pour les mots féminins masculins donc je connaissais pas si euh à quoi il ressemble par exemple $(2015, \mathrm{RZ})$

Ici, on constate aussi la présence d'un marqueur métadiscursif je sais pas dans le même énoncé. En utilisant ces trois éléments, le locuteur invite son allocutaire à partager son opinion sur ce qui est dit.

\subsubsection{Marqueur de concession}

Très souvent, dans ce cas-là, on voit deux arguments en utilisant des connecteurs argumentatifs, tels que mais, quand même, etc.

(13) donc euh jusqu'à ce moment je parle pas très bien le français mais je lis bien oui je lire les romans quoi (2014, WHD)

Il est clair qu'ici le locuteur a fait deux arguments concernant sa compétence linguistique, qui s'opposent : je parle pas très bien le français et je lis bien. En utilisant le connecteur mais, le locuteur met clairement plus de poids sur le deuxième argument. L'emploi de quoi à la fin du deuxième argument invite son allocutaire à se positionner par rapport à ce qui est dit. 
Pour conclure cette partie, on dirait que les fonctions discursives de quoi sont assez variées dans le discours des locuteurs non-natifs. On constate que ces locuteurs non seulement apprennent à l'utiliser comme marqueur discursif mais aussi sous des contextes différents. Il faut, cependant, admettre que les contextes sont plus complexes et variés dans le discours des locuteurs natifs. Par exemple, les types de quantificateurs s'utilisent avec le marqueur discursif quoi sont évidemment beaucoup moins nombreux dans la production des locuteurs non-natifs. Cela pourrait être dû au nombre très limité de quoi en total dans notre corpus. On pourrait conclure que même si les locuteurs non-natifs maîtrisent l'emploi de quoi sous des contextes discursifs différents, la fréquence d'emploi est quand même beaucoup plus base.

\subsection{Facteurs extralinguistiques}

Comme l'évoqué plus haut, plusieurs facteurs sociaux ont été prouvés significatifs pour l'emploi de quoi dans le discours des locuteurs natifs. Dans cette partie, nous essayons d'examiner si ces facteurs extralinguistiques influencent aussi la production de quoi chez les locuteurs non-natifs. Les facteurs non-linguistiques qu'examinons nous sont : le sexe, la durée de séjour et le statut professionnel du locuteur.

\subsubsection{Sexe}

Comme nous avons déjà montré dans la partie précédente, le sexe du locuteur est un facteur significatif pour les locuteurs natifs. C'est un marqueur très sexe-orienté.

Tableau 1. Distribution de MD quoi dans les deux groupes de sexe

\begin{tabular}{|c|c|l|l|}
\hline Sexe & $\begin{array}{c}\text { Nombre } \\
\text { de } \\
\text { locuteurs }\end{array}$ & Quoi & $\%$ \\
\hline Féminin & 22 & 18 & $10.9 \%$ \\
\hline Masculin & 15 & 147 & $89.1 \%$ \\
\hline Total & 37 & 165 & $100 \%$ \\
\hline
\end{tabular}

On constate que le nombre des locuteurs féminins est un peu plus que le nombre des locuteurs masculins. Cependant, les locuteurs masculins, selon tableau 1, produisent beaucoup plus d'occurrences de quoi que les locuteurs féminins. Le nombre inégal des deux sexes n'impacte pas le résultat final obtenu concernant ce facteur. Il semble que les femmes évitent l'emploi de ce marqueur comme ce que fait les locuteurs natifs.

Il existe deux interprétations : soit les femmes perçoivent la tendance chez les locuteurs natifs et évitent intentionnellement l'usage de ce marqueur ; soit les femmes ne sont pas assez exposées à ce marqueur discursif et n'acquièrent pas son emploi. La première serait plus plausible si ces locuteurs sont des locuteurs avancés et s'elles sont des usagers fréquents du français quotidiennement. Souvent, les locuteurs avancés perçoivent bien des marqueurs sociaux et commencent à varier leur usage langagier en fonction des contextes différents et des registres différents, tandis que les locuteurs débutant ou intermédiaire se concentrent plutôt sur des normes prescrites, puisqu'ils ne maîtrisent pas encore très bien les règles basiques de la langue et sont donc peu probables de tenter à varier leur usage langagier. Mais comme le niveau linguistique de nos locuteurs sont inégaux (surtout certains locuteurs viennent d'arriver en 
France), il est peu probable que ce soit le cas. En ce qui concernent la deuxième interprétation, ça dépend de beaucoup d'autres facteurs non-linguistiques : la durée de séjour en France, le type de réseau social, la fréquence d'usage du français journalier, l'activité extracurriculaire, etc. Sans une enquête plus profonde et plus complète sur ces locuteurs, il est impossible à ce stade de décider si cela est le cas.

\subsubsection{Durée de séjour en France}

Comme souligné dans beaucoup d'autres recherches, la durée de séjour pourrait être significative pour l'apprentissage des formes non-standards, tel que l'omission de ne dans la négation. L'apprentissage des marqueurs discursifs demande du temps passé dans l'environnement naturel de la langue cible, puisque souvent ces formes ne sont pas enseignées explicitement au sein de la classe de la langue. Pour notre recherche actuelle, nous avons divisé nos locuteurs en trois groupes en fonction de leur durée de séjour en France : 1-3 ans, 4-6 ans et plus de 7 ans. Notre hypothèse est que le plus long on reste dans la communauté le plus on utilise quoi.

Tableau 2. Distribution de MD quoi dans les groupes de durée de séjour en France

\begin{tabular}{|c|l|l|l|}
\hline $\begin{array}{c}\text { Durée } \\
\text { de } \\
\text { séjour } \\
\text { (année) }\end{array}$ & $\begin{array}{c}\text { Nombre } \\
\text { de } \\
\text { locuteurs }\end{array}$ & Quoi & $\%$ \\
\hline $1-3$ & 17 & 36 & $21.82 \%$ \\
\hline $4-6$ & 10 & 18 & $10.91 \%$ \\
\hline$>7$ & 10 & 111 & $67.27 \%$ \\
\hline
\end{tabular}

Tableau 2 nous montre que les locuteurs qui ont passé plus de sept ans en France utilisent beaucoup plus fréquemment le quoi que les locuteurs dans les deux autres groupes comme prévu. Cela indique qu'une plus longue durée de séjour favoriserait l'apprentissage d'emploi de ce marqueur discursif, puisque le taux d'emploi pour le troisième groupe est significativement plus haut que les deux autres groupes. Cependant, on constate un plus haut pourcentage chez le premier group, le groupe des nouveaux-arrivants, que chez le deuxième groupe. Cela suggère que l'apprentissage de ce marqueur discursif pourrait suivre ce qu'on appelle «U-shaped development ». C'est-à-dire qu'au début, soit ils ont appris cette forme comme une locution figée, soit ils l'utilisent sous l'influence du transfert de leur langue maternelle. Pour vérifier si cela est de la conséquence d'un transfert linguistique, il faut regarder le marqueur discursif correspondant dans leur langue maternelle.

\subsubsection{Statut professionnel}

Le dernier facteur que nous voudrions examiner ici est le statut professionnel. Nous les avons classés dans trois groupes : les étudiants, les professionnels et les sans-travail. La raison pour le choix d'examiner ce facteur est que ces trois statuts leur donnent des accès différents au français : le registre, la fréquence du contact avec les locuteurs natifs, la fréquence d'utiliser le français, etc. Comme le marqueur discursif est employé le plus dans le registre formel et que le marqueur discursif quoi est fortement socialement marqué, il est peu possible qu'on trouve son 
emploi dans le discours des professeurs à l'université. Cependant, entre les collègues au travail, la probabilité augmente. Notre hypothèse est que ceux qui travaillent utilisent plus ce marqueur que les étudiants.

Tableau 3. Distribution de MD quoi dans les groupes de profession

\begin{tabular}{|c|c|l|l|}
\hline $\begin{array}{c}\text { Statut } \\
\text { professionnel }\end{array}$ & $\begin{array}{c}\text { Nombre } \\
\text { de } \\
\text { locuteurs }\end{array}$ & Quoi & $\%$ \\
\hline Étudiant & 20 & 19 & $11.52 \%$ \\
\hline Professionnel & 16 & 144 & $87.27 \%$ \\
\hline$/$ & 1 & 2 & $1.21 \%$ \\
\hline
\end{tabular}

Il semble que, selon le tableau 3, la profession a clairement une influence sur l'emploi de ce marqueur discursif. Ceux qui travaillent l'utilisent beaucoup plus que ceux qui ne mènent pas de vie professionnelle. Cela prouve qu'une interaction fréquente avec les locuteurs natifs favorise l'apprentissage de ce marqueur. Les étudiants, même ils ont des camarades français à l'école, ils n'interagissent pas forcément avec eux, puisque leur cursus de formation leur demande simplement de suivre des séminaires où le professeur parle pour la plupart du temps en utilisant un registre plutôt formel qui ne facilite pas l'emploi des marqueurs informels ou socialement marqués tels que quoi.

Puisque nous n'avons eu qu'un seul locuteur dans le troisième groupe, nous n'avons pas pu comparer le résultat de ce groupe avec le résultat des deux autres groupes. Cependant, il faut dire qu'il serait intéressant à regarder de plus près des locuteurs de ce groupe dans la future. En fait, le seul locuteur dans ce groupe est une femme au foyer dont le mari est Français. D'une part, son mari va utiliser un registre informel avec elle à la maison, elle aura beaucoup plus d'accès à un français non-standard que les autres locuteurs dans les deux autres groupes; d'une autre part, puisqu'elle ne travaille pas et qu'elle ne connaît pas beaucoup d'amis ici en France, (surtout le mari travaille toute la journée et ne rentre à la maison que le soir) elle aura dans ce sens-là moins d'opportunité à pratiquer le français que les professionnels ou même que les étudiants. Cela nous intrigue à demander si les femmes au foyer utilisent plus ou moins le marqueur quoi dans leur production orale?

Pour conclure cette partie, comme ce qui est observé chez les locuteurs natifs, parmi les locuteurs non-natifs, les hommes utilisent beaucoup plus de quoi que les femmes. La durée de séjour et le statut professionnel semblent aussi significatifs pour son emploi dans notre étude. Cependant, en raison d'un nombre très limité des occurrences exploitables de quoi trouvées dans notre corpus, il est impossible à ce stade de donner des interprétations conclusives. Les présents résultats ne nous servent que des guides pour les futures études.

\section{Conclusion}

Dans cet article, nous avons comparé l'usage du marqueur discursif quoi dans le discours des locuteurs natifs et non-natifs du français en mettant un accent sur les divers contextes de sa présence. Nous avons également examiné comment les facteurs extralinguistiques influencent son emploi chez les non-natifs. Les exemples ont été tirés de notre propre corpus construit en 2014. Ils sont tous issus des conversations semi-dirigées suivant le protocole des entretiens sociolinguistiques qui facilitent la présence des marqueurs discursifs. 
On constate que les locuteurs non-natifs utilisent le marqueur discursif quoi beaucoup moins fréquemment que les locuteurs non-natifs. Cependant, ils utilisent quoi comme marqueur discursif sous des contextes divers comme font les locuteurs natifs tout en tenant compte que les contextes sont plus complexes et variés dans le discours des locuteurs natifs.

En ce qui concerne les facteurs extralinguistiques, les hommes utilisent beaucoup plus de quoi que les femmes. La durée de séjour et le statut professionnel semblent aussi significatifs pour son emploi dans notre étude. En raison d'un nombre très limité des occurrences exploitables de quoi trouvées dans notre corpus, il est impossible à ce stade de donner des interprétations conclusives.

Le travail actuel présente et complète partiellement la documentation des emplois des marqueurs discursifs dans le discours des locuteurs non-natifs. Les marqueurs discursifs méritent notre attention particulière non seulement dans le discours des locuteurs natifs mais aussi dans le discours des locuteurs non-natifs. Puisque les marqueurs discursifs pourraient être un très bon indice de la maîtrise de la langue cible, il serait mieux de les enseigner au sein de la classe des langues secondes/étrangères au lieu de les traiter comme mal parler ou de les négliger. Là, comment préparer les apprenants à une éventuelle stigmatisation de certaines façons de parler qui, en fonction de la communication situative, s'adaptent plus ou moins bien au registre attendu devient un enjeu permanent dans la pratique pédagogique. Il faut toujours poser la question: Qu'est-ce que l'on enseigne ? Comment on enseigne? Mais surtout il faut se demander: Pourquoi on enseigne? Souvent, on souligne l'important d'un français standard conformé tout à fait aux normes prescrites par les méthodes grammaticales dans la classe des langues. Cependant, une fois sortis de la classe, les élèves seraient bouleversés par le français qu'ils entendent, parce qu'ils rencontrent des usages qui ne pourraient pas être expliqués par ce qu'ils apprennent à l'école. Le problème est que très souvent les apprenants ne savent pas adapter leur emploi langagier au registre. Une initiation de la différenciation entre le français standard et le français journalier dans le cursus serait beaucoup recommandée.

En ce qui concerne les marqueurs discursifs, il est possible que les différents marqueurs discursifs apparaissent dans des différents stades d'études des locuteurs non-natifs tout au long de leur apprentissage. Il sera donc intéressant de voir l'ordre, s'il en existe un, suivant lequel les locuteurs non-natifs apprennent les marqueurs discursifs dans une situation naturelle. Aussi, dans une situation non-naturelle, par exemple, au sein de la classe des langues dans un endroit où le français n'est pas la langue de pratique, est-ce que les locuteurs non-natifs utilisent aussi les marqueurs discursifs et si oui dans quelle mesure?

Pour le futur travail, nous suggérons de voir comment l'usage des marqueurs discursifs évoluent chez les locuteurs natifs du français et les cas plus complets de la co-variation des marqueurs discursifs dans le discours des locuteurs natifs ainsi que dans le discours des locuteurs non-natifs.

\section{Références bibliographiques}

Andersen, H. L. (2007). Marqueurs discursifs propositionnels. Langue française, (2), 13-28.

Beeching, K. (2002). Gender, politeness and pragmatic particles in French (No. 104). John Benjamins Publishing.

Beeching, K. (2007). La co-variation des marqueurs discursifs bon, c'est-à-dire, enfin, hein, quand même, quoi et si vous voulez: une question d'identité?. Langue française, (2), 78-93.

Blakemore, D. (1987). Semantic constraints on relevance.

Chanet, C. (2001). 1700 occurrences de la particule quoi en français parlé contemporain : approche de la « distribution » et des fonctions en discours. Marges linguistiques, 2, 56-80.

Chanet, C. (2004). Fréquence des marqueurs discursifs en français parlé : quelques problèmes de méthodologie. Recherches sur le français parlé, 18(83), 106. 
Dostie, G. (2004). Pragmaticalisation et marqueurs discursifs.

Erman, B. (2001). Pragmatic markers revisited with a focus on you know in adult and adolescent talk. Journal of pragmatics, 33(9), 1337-1359.

Fernandez, M. J. (1994). Les particules énonciatives dans la construction du discours. Linguistique nouvelle.

Fraser, B. (1999). What are discourse markers?. Journal of pragmatics, 31(7), 931-952.

Hansen, M. B. M. (1997). Alors and donc in spoken French: A reanalysis. Journal of Pragmatics, 28(2), 153-187.

Jucker, A. H., \& Ziv, Y. (Eds.). (1998). Discourse markers: Descriptions and theory (Vol. 57). John Benjamins Publishing.

Lefeuvre, F. (2006). Quoi de neuf sur « quoi »?: étude morphosyntaxique du mot « quoi ». Presses Univ. de Rennes.

Lefeuvre, F. (2011). Bon et quoi à l'oral: marqueurs d'ouverture et de fermeture d'unités syntaxiques à l'oral. Linx. Revue des linguistes de l'université Paris X Nanterre, (64-65), 223-240.

Redeker, G. (1990). Ideational and pragmatic markers of discourse structure. Journal of pragmatics, 14(3), 367-381.

Redeker, G. (1991). Linguistic markers of discourse structure. Linguistics, 29(6), 1139-1172.

Schiffrin, D. (1988). Discourse markers (No. 5). Cambridge University Press.

Schourup, L. C. (1985). Common Discourse Particles in English Conversation: like, well, y'know, NY: Garland. 